\title{
HST/STIS imaging of Fomalhaut: New main belt structure and confirmation of Fomalhaut $b$ 's eccentric orbit
}

\author{
P. Kalas ${ }^{1}$, J. R. Graham ${ }^{1}$, M. P. Fitzgerald ${ }^{2}$ and M. Clampin ${ }^{3}$ \\ ${ }^{1}$ Astronomy Department, University of California, Berkeley, CA 94720 \\ email: kalas@berkeley.edu \\ ${ }^{2}$ Dept. of Physics and Astronomy, UCLA, Los Angeles, CA \\ ${ }^{3}$ NASA Goddard Space Flight Center, Greenbelt, MD
}

\begin{abstract}
High contrast imaging observations with the Hubble Space Telescope show that the nearby star Fomalhaut is surrounded by a dusty debris belt and a candidate planet, Fomalhaut $b$, located just inside the edge of the belt. Fomalhaut $b$ has unexpected characteristics, such as a relatively blue spectrum, leading to the hypothesis that the detected object is a low-mass planet hosting a giant planetary dust ring or cloud seen in reflected light. Here we present new HST/STIS observations made in 2010 and 2012 that authenticate the existence of Fomalhaut $b$. Our MCMC analysis of four epochs of astrometry spread over eight years indicate that the orbit has $a \sim 170 \mathrm{AU}$ and $e \sim 0.85$. Fomalhaut $b$ 's orbit is apsidally aligned with the main belt, and periapse is located approximately $\sim 30 \mathrm{AU}$ south of the star. We also show the existence of $\mathrm{a} \sim 50 \mathrm{AU}$ wide azimuthal dust depletion in the dust belt. These new findings provide a revised picture of Fomalhaut as a dynamically complex system, where the orbit of Fomalhaut $b$ and the belt structure signify the presence of additional massive planets orbiting the star.
\end{abstract}

Keywords. planets \& satellites: individual: Fomalhaut $b$

Background: As of this writing, the existence of Fomalhaut $b$ has been confirmed by three epochs of observations with the Hubble Space Telescope (HST) between 2004 and 2010, in work published by three independent research groups (Kalas et al. 2008, Kalas et al. 2010, Kalas 2011, Currie et al. 2012, Galicher et al. 2013). Though confirmed as a real, low-mass, common proper motion companion to the A3V star Fomalhaut ( $\alpha$ PsA; HD 216956), Fomalhaut $b$ has surprising observational properties. One of these surprises was that the third epoch of observation made in 2010 showed that Fomalhaut b's sky plane motion was directed toward instead of parallel to the dust belt's inner edge. This motion implied a highly eccentric orbit. However, Kalas et al. (2010) \& Kalas (2011) argued that this required observational confirmation because the third epoch represented a change of instrumentation from HST/ACS to HST/STIS. Thus there were potential problems concerning systematic errors in the relative astrometry that could be introduced by the changeover in imaging cameras. Here we report that the fourth epoch detection of Fomalhaut $b$, made with HST/STIS in 2012, con firms that Fomalhaut $b$ has a highly eccentric orbit. For detailed information we refer the reader to Kalas et al. (2013).

HST/STIS Observations \& Results: Figure 1 shows our 2012 detection of Fomalhaut's dust belt and Fomalhaut $b$ using HST/STIS. STIS consists of a $1024 \times 1024$ pixel (0.0407" / pixel) CCD operating between $0.20 \mu \mathrm{m}$ and $1.03 \mu \mathrm{m}$, with non-transparent focal plane, wedge-shaped masks to prevent saturation of bright stars, but without a pupil plane mask to suppress diffraction around the secondary support spiders. The bright PSF halo and diffraction spikes are subtracted by angular differential imaging - the telescope 
is rotated to 12 different orientations each separated by $2^{\circ}$ about the optical axis in 12 different orbits. One of the key problems for relative astrometry is ascertaining the position of Fomalhaut A (the central star) behind the occulting wedges. We used a variety of techniques to estimate this position, such as by using the diffraction spikes to provide one estimate of the position. We also re-analysed the astrometry for the 2004 and 2006 observations made with HST/ACS, and the 2010 observations made with HST/STIS. Other sources of astrometric error, such as uncorrected geometric distortion and guide star tracking drift, were also estimated using a variety of techniques. A detailed list of astrometric error sources, and the four epochs of astrometry with their associated error bars, are provided in Kalas et al. (2013).

Figure 1 illustrates three of the key new findings made with the HST/STIS images. First, the astrometry confirms that the sky-plane motion of Fomalhaut $b$ is directed to the

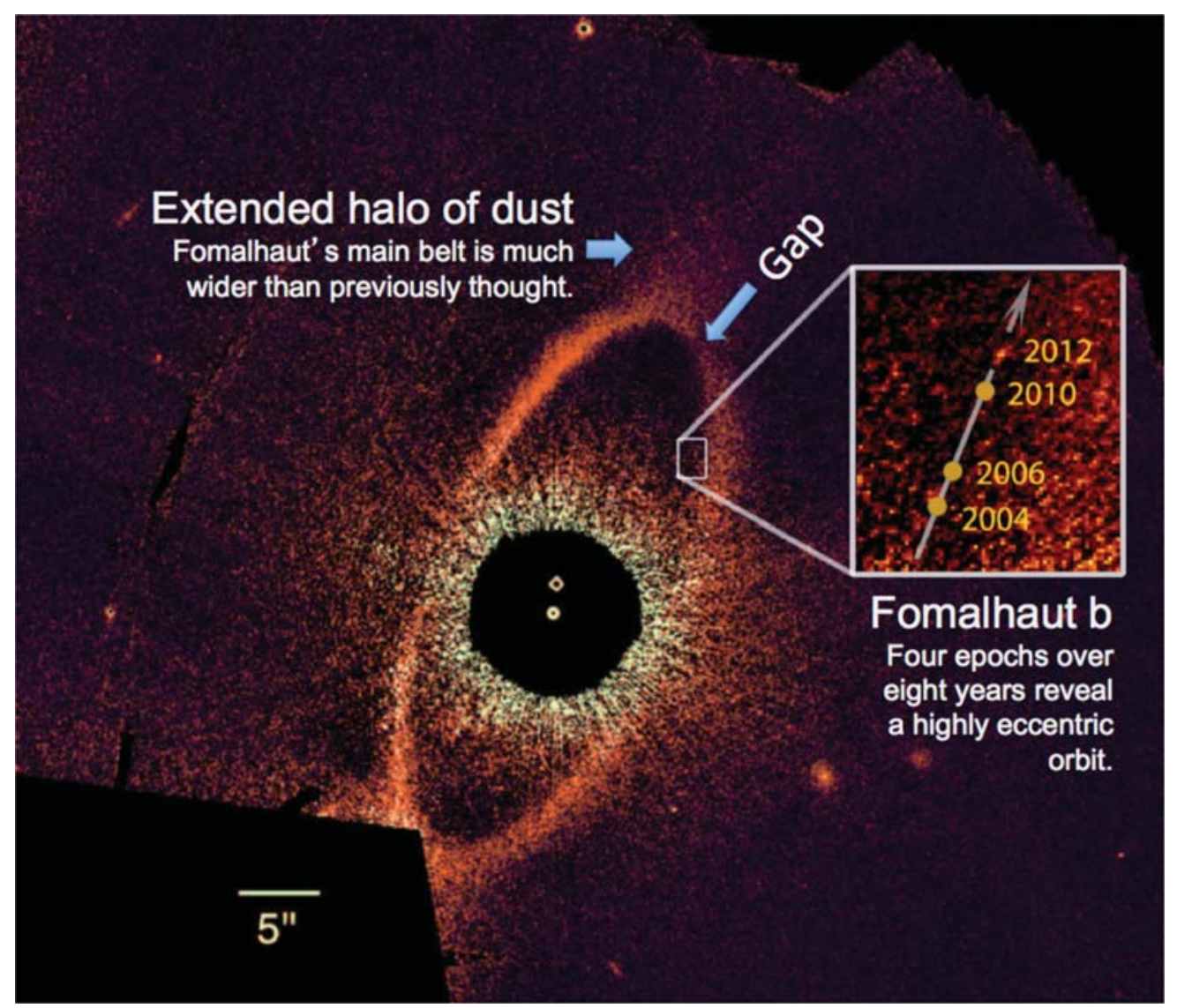

Figure 1. Detections of Fomalhaut $b$ and structure in Fomalhaut's dust belt made in 2010 \& 2012 with optical HST/STIS observations (Kalas et al. 2013). North is up, east is left, and $5^{\prime \prime}$ corresponds to $39 \mathrm{AU}$. The box inset illustrates the positions of Fomalhaut $b$ over eight years, with a trajectory that is northwestward and will appear to cross the belt over the next few decades. Periastron is south of the star at $30 \pm 24 \mathrm{AU}$. The circle marks the position of the star, and the diamond marks the geometric center of the belt that is offset from the star by $\sim 13 \mathrm{AU}$ in the sky plane. The main belt has a tenuous halo of nebulosity detected to the northwest suggesting that dust grains extend to at least 209 AU radius. An additional dust belt feature discovered in the 2010 STIS observations and confirmed in the 2012 data is an azimuthal gap at $\mathrm{PA}=331^{\circ}$. In the deprojected reference frame, the gap can be characterized as a $50 \%$ decrease in scattered light with $\mathrm{FWHM} \approx 50 \mathrm{AU}$. 
northwest in a path that in projection crosses the dust belt. Second, the northwest side of the belt shows a tenuous nebulosity indicating that the dust seen in optical scattered light is extended to at least 209 AU radius. Third, the dust belt surface brightness in the azimuthal direction diminishes at $\mathrm{PA}=331^{\circ}$. In a deprojected view, the azimuthal belt gap has FWHM 50 AU.

The 1- $\sigma$ errors on the astrometry of Fomalhaut $b$ relative to the star are approximately 20 mas for the 2004 and 2006 HST/ACS data, and 40 mas for the 2010 and 2012 HST/STIS data. We developed an MCMC method to sample the posterior probability distribution for orbital elements based on the astrometry, yielding median and rms values of $a=170 \pm 30 \mathrm{AU}, e=0.85 \pm 0.07$ (assuming $M_{\star}=1.92 \pm M_{\odot} ;$ Mamajek 2012).

The orbit of Fomalhaut $b$ is confirmed to be highly eccentric. This puts periastron slightly over a century ago in the region a few tens of AU from the star, and in the sky plane projection Fomalhaut $b$ appears to cross the belt over the next few decades. The median inclination of the orbital plane $\left(-56^{\circ}\right)$ is close to that of the belt plane $\left(-66^{\circ}\right)$ and apsidal alignment between Fomalhaut $b$ and the belt is indicated, within the uncertainties. Overall, additional astrometric points in future work are required to determine if Fomalhaut $b$ is on a collision course with the main belt, or if it will pass above or below the belt over the next few decades.

Implications of Fomalhaut $b$ 's highly eccentric orbit: The dynamical history of the Fomalhaut system is clearly more complex than previously thought. Kalas et al. (2005) presented detailed measurements of the dust belt's stellocentric offset and sharp inner edge consistent with the hypothetical secular perturbations of an undetected planet. When the existence of Fomalhaut $b$ was subsequently discovered as lying just 18 AU interior to the dust belt, it naturally became the top candidate for explaining these belt properties (Kalas et al. 2008, Chiang et al. 2009). However, Fomalhaut b's high eccentricity now complicates the dynamical picture because: (1) a mechanism, such as planet-planet scattering by a more massive Fomalhaut $c$, is required to explain Fomalhaut $b$ 's high eccentricity, and (2) the stellocentric and sharp inner edge of the dust belt also require a yet-to-be-detected planet on a relatively low eccentricity orbit.

Fomalhaut $b$ 's origin and physical nature also continue to be open topics for additional research. Kalas et al. (2008) argued that because Fomalhaut $b$ is detected in the optical and not in the infrared, the optical flux could represent light scattered from a circumplanetary ring system. Kennedy \& Wyatt (2011) studied the possibility that instead of a circumplanetary dust ring, a circumplanetary dust cloud could be generated by the collisional erosion of irregular satellites. The third possibility, that Fomalhaut $b$ is a dust clump resulting from a collision between two planetesimals, was considered by Kalas et al. (2008), Currie et al. (2012) and Galicher et al. (2013) and generally thought to be unlikely, though not ruled out. Kalas et al. (2013) argue that the periastron distance of $\sim 30$ AU decreases the previously assumed Hill radius for Fomalhaut $b$ $\left(R_{H}=a_{p l}(1-e)\left(m_{p l} / 3 M_{\star}\right)^{1 / 3}\right)$. They establish through a numerical experiment that Fomalhaut $b$ needs a few times a Ceres mass to maintain a long lived cloud of dust from irregular satellites, as in the Kennedy \& Wyatt (2011) model.

For the maximum mass of Fomalhaut $b$, the infrared non-detection suggest $\lesssim 1$ Jupiter mass based on models of planet luminosity evolution (Kalas et al. 2008, Janson et al. 2012, Kenworthy et al. 2013). A complementary, model-dependent mass constraint derives from the dynamics of the system. Kalas et al. (2013) numerically studied cases where a Jupiter mass Fomalhaut $c$ is nested within and coplanar with the dust belt, with $a=120 \mathrm{AU}$, $e=0.1$, and Fomalhaut $b$ is a planet with masses between Earth and Jupiter that has a highly eccentric coplanar or non-coplanar orbit (relative to Fomalhaut $c$ and the dust 
belt). The coplanar cases are relatively short-lived (i.e. the orbit of Fomalhaut $b$ changes on $\sim 10^{6}$ yr timescales). A coplanar, Saturn-mass Fomalhaut $b$ erodes the belt edges on $10^{5}$ year timescales (the belt spreads radially), but a coplanar, Neptune-mass has an insignificant effect on that timescale. In the non-coplanar simulations, the ascending and descending nodes of Fomalhaut $b$ may lie significantly within or beyond the perimeter of the belt and Fomalhaut $c$, with negligible dynamical effect on $\sim 10^{6}$ yr timescales. However, secular perturbations are possible on $10^{7}-10^{8}$ yr timescales that modify the orbit of Fomalhaut $b$. Secular perturbations can reduce Fomalhaut $b$ 's $a$ and $e$ such that its apastron occurs within the main belt. Fomalhaut $b$ therefore transitions into a significant perturber of the belt. Alternately, secular perturbations could increase Fomalhaut $b$ 's $a$ and $e$ such that its perturbations on the system transition to longer timescales.

To summarize, a variety of factors and assumptions underly any determination of Fomalhaut $b$ 's mass and origin from dynamics alone. Future work should be dedicated to detecting the hypothetical Fomalhaut $c$. Moreover, Fomalhaut $b$ could be directly detected at sub-mm wavelengths with ALMA if in fact the circumplanetary dust cloud or ring hypothesis is correct. The azimuthal gap shown in Fig. 1 is also unexplained as of this writing - Fomalhaut $b$ 's orbit does not cross this region. Over a decade timescale, the $0.4^{\prime \prime}$ sky plane rotation of the gap is potentially observable with ALMA.

Acknowledgements: We thank GO-11818 \& GO-12576 provided by NASA from STScI; NSF AST-0909188; NASA Origins 09-SSO09-0124; and Univ. Calif. LFRP-118057.

\section{References}

Chiang, E., Kite, E., Kalas, P., Graham, J. R. \& Clampin, M. 2009, ApJ, 693, 734

Currie, T., Debes, J., Rodigas, T. J., et al. 2012, ApJL, 760, L32

Galicher, R., Marois, C., Zuckerman, B., \& Macintosh, B. 2013, ApJ, 769, 42

Janson, M., Carson, J. C., Lafrenière, D., et al. 2012, ApJ, 747, 116

Kalas, P., Graham, J. R., \& Clampin 2005, Nature, 435, 1067

Kalas, P., Graham, J. R., Chiang, E., et al. 2008, Science, 322, 1345

Kalas, P., Graham, J. R., Fitzgerald, M., \& Clampin, M. 2010, in In the Spirit of Lyot 2010, ed. A. Boccaletti.

Kalas, P. 2011, in The Astrophysics of Planetary Systems, IAU Symposium, Vol. 276, p. 279.

Kalas, P., Graham, J. R., Fitzgerald, M., \& Clampin, M. 2013, in ApJ, 775, 56

Kennedy, G. M. \& Wyatt, M. C. 2011, MNRAS, 412, 2137

Kenworthy, M. A., Meshkat, T., Quanz, S. P., et al. 2013, ApJ, 764, 7

Mamajek, E. E. 2012, ApJL, 754, L20

\section{Discussion}

ERIC GAIDOs: Photometric variability was reported in the discovery paper of Fomalhaut $b$. What is the status of this with the new observations?

Paul Kalas: Between 2010 and 2012 we do not detect photometric variability in the STIS detections of Fomalhaut $b$. In our tests using injected point sources, we find that the photometric uncertainty due to speckle noise is $\sim 35 \%$. These tests also show that an injected point source may gain an artificial, extended morphology due to the noise. With respect to the first two epochs of discovery images made with HST/ACS, other groups report somewhat conflicting photometry that illustrate the significant role of using different data reduction techniques and measurement methods. The issue of photometric variability and extended morphology therefore require additional future work. 